JOURNAL CLUB

\title{
Ambrisentan for pulmonary fibrosis
}

This randomised, double-blind, placebo-controlled study is one of the largest multicentre studies investigating whether ambrisentan (an endothelin A receptor antagonist) reduces the rate of idiopathic pulmonary fibrosis (IPF) progression.

The investigators compared ambrisentan (more commonly used in the treatment of pulmonary hypertension $(\mathrm{PH})$ ) with placebo on the basis of preclinical studies suggesting endothelin receptor blockade decreased the severity of pulmonary fibrosis.

The primary endpoint was time to IPF progression defined as either death, respiratory hospitalisation or prespecified decrease in lung function (whichever came first).

Target recruitment was 660 over 18 months. However, at $75 \%$ of enrolment (492) the study was terminated due to a low likelihood of showing efficacy by the study end.

However, more worryingly ambrisentan-treated patients were more likely to have disease progression, more likely to suffer lung function decline, more likely to suffer respiratory hospitalisations and more likely to die when compared with placebo.

One reason given for the worse outcomes is worse baseline IPF severity. However, even allowing for this does not fully explain the differences seen. In addition, they used patients with $<5 \%$ honeycombing on CT, which would suggest less severe disease, and also therefore less likely to have $\mathrm{PH}$ (approximately $10 \%$ in each group as determined by right heart catheterisation), a patient group which one may speculate would do better with ambrisentan.

The authors concluded that ambrisentan was not effective in treating IPF and may be associated with an increased risk for disease progression and hospitalisation.

- Raghu G, Behr J, Brown KK, et al. Treatment of idiopathic pulmonary fibrosis with ambrisentan a parallel, randomized trial. Ann Intern Med 2013;158:641-9.

\section{Ravi Punwani}

Correspondence to Dr Ravi Punwani, The Royal Marsden NHS Foundation Trust, Down's Road, Sutton, Surrey SM2 5PT, UK; ravi.punwani@rmh.nhs.uk

Thorax 2014;69:489. doi:10.1136/thoraxjnl-2013-204438 